\title{
Exploring the multifaceted circumstellar environment of the Luminous Blue Variable HR Carinae
}

\author{
C. S. Buemi ${ }^{1 \star}$, C. Trigilio ${ }^{1}$, P. Leto ${ }^{1}$, G. Umana ${ }^{1}$, A. Ingallinera ${ }^{1}$, F. Cavallaro ${ }^{1,2,3}$, \\ L. Cerrigone ${ }^{4}$, C. Agliozzo ${ }^{5,6}$, F. Bufano ${ }^{1}$, S. Riggi ${ }^{1}$, S. Molinari ${ }^{7}$, F. Schillirò ${ }^{1}$ \\ ${ }^{1}$ INAF - Osservatorio Astrofisico di Catania, Via S. Sofia 78, 95123 Catania, Italy \\ ${ }^{2}$ CSIRO Astronomy and Space Science, PO Box 76, Epping, NSW 1710, Australia \\ ${ }^{3}$ Università di Catania, Dipartimento di Fisica e Astronomia, Via Santa Sofia, 64, 95123 Catania, Italy \\ ${ }^{4}$ ASTRON, the Netherlands Institute for Radioastronomy, PO Box 2, 7990 AA Dwingeloo, The Netherlands \\ ${ }^{5}$ Millennium Institute of Astrophysics, Santiago 7500011, Chile \\ ${ }^{6}$ Universidad Andrés Bello, Avda. Republica 252, Santiago 8320000, Chile \\ ${ }^{7}$ INAF-Istituto di Astrofisica e Planetologia Spaziale, via Fosso del Cavaliere 100, 00133 Roma, Italy
}

\begin{abstract}
We present a multi-wavelength study of the Galactic Luminous Blue Variable HR Carinae, based on new high resolution mid-infrared (IR) and radio images obtained with the Very Large Telescope (VLT) and the Australia Telescope Compact Array (ATCA), which have been complemented by far-infrared Herschel-PACS observations and ATCA archive data. The Herschel images reveal the large-scale distribution of the dusty emitting nebula, which extends mainly to the North-East direction, up to 70 arcsec from the central star, and is oriented along the direction of the space motion of the star. In the mid-infrared images, the brightness distribution is characterized by two arc-shaped structures, tracing an inner envelope surrounding the central star more closely. At radio wavelengths, the ionized gas emission lies on the opposite side of the cold dust with respect to the position of the star, as if the ionized front was confined by the surrounding medium in the North-South direction. The comparison with previous data indicates significant changes in the radio nebula morphology and in the massloss rate from the central star, which has increased from $6.1 \times 10^{-6} \mathrm{M}_{\odot} \mathrm{yr}^{-1}$ in 1994-1995 to $1.17 \times 10^{-5} \mathrm{M}_{\odot} \mathrm{yr}^{-1}$ in 2014 . We investigate possible scenarios that could have generated the complex circumstellar environment revealed by our multi-wavelength data.
\end{abstract}

Key words: circumstellar matter - stars: early-type - stars: individual (HR CAR) - stars: winds, outflows -radio continuum: stars - infrared: stars

\section{INTRODUCTION}

In the traditional view, Luminous blue variables (LBVs) are a class of massive and very luminous stars in the post mainsequence evolution stage, moving from the hot supergiant towards the Wolf-Rayet (WR) phase (Humphreys \& Davidson 1994; Lamers \& Nugis 2002). Currently, their link to other advanced evolutionary phases of massive stars, such as supernovae, represents a hot topic of discussion (Barlow et al. 2005; Kotak \& Vink 2006). They are among the rarest types of stars because of the relatively shortness of this evolutionary phase, believed to last about $10^{4}-10^{5}$ $\mathrm{yr}$, and the high initial masses, greater than $22 \mathrm{M}_{\odot}$ (Lamers et al. 2001). Despite heir rarity, LBVs may play an important role in the chemical enrichment of the interstellar medium, due to the large amount of processed material injected by means of continuous winds and intense eruptions. During their short lives, LBVs

^E-mail: cbuemi@ oact.inaf.it undergo heavy episodic mass-loss events during which they lose a large fraction of their H-rich outer layers, as evidenced by the observations of their complex circumstellar environments. The study of the geometrical and physical properties of these stellar ejecta could provide us with important insights on the mass-loss phenomenon and the evolution of the progenitor star. The richness of their morphological structures suggests that the mechanisms that create and shape the extended nebulae are several and still poorly understood. Most proposed models are related to hydrodynamic explosions and continuum-driven super-Eddington winds, rather than line-driven stellar winds (Humphreys \& Davidson 1994; Smith \& Owocki 2006; Smith 2014). There is a growing evidence for bipolar morphology occurring frequently in LBV nebulae (White 2000; Weis 2001; Umana et al. 2005), and several models have been proposed to reproduce such structures, most of them considering the shells as the result of the interaction between a spherical symmetric stellar wind and an equatorial disc (Nota et al. 1995; Ohara et al. 2003). However, it is unclear whether the bipolar na- 
ture of the LBV ejecta is due to a pre-existing density contrast or the wind itself is asymmetric (Frank et al. 1998; Maeder \& Desjacques 2001). For LBVs with ring nebulae, equatorial mass-loss (enhanced by the fast rotation of the star, the presence of a companion star, or a magnetic field) is often proposed (see Gvaramadze et al. 2015, and references therein).

Recently, the Herschel and Spitzer space missions allowed for a very detailed mapping of the circumstellar dusty environments associated with Galactic LBVs. At the same time, high angular resolution radio observations offer the additional possibility to reveal the ionized gas inside the dusty envelope. This provides useful clues to understand the interaction between the ejecta and the surrounding medium. The synergistic use of different techniques at different wavelengths is necessary for a good understanding of the physical conditions in LBVs, allowing us to analyse the different emitting components that coexist in the stellar ejecta. In some cases, evidence for asymmetric mass loss (Buemi et al. 2010) and possible mutual interaction between gas and dust components (Umana et al. 2010) has emerged from the comparison of the midIR and radio images of the nebulae, while important information on the gas and dust composition has been derived from the Spitzer and Herschel spectra (Umana et al. 2009; Vamvatira-Nakou et al. 2013, 2015).

HR Car is a member of the small group of confirmed galactic LBVs, showing the typical characteristic of photometric and spectroscopic S Doradus type variability (van Genderen 2001). The central star, classified as B2I, has a luminosity of $5 \times 10^{5} \mathrm{~L} \odot$, as derived from spectral analysis of the Balmer lines performed by Machado et al. (2002), and is located at a distance of $5.4 \pm 0.1 \mathrm{kpc}$ (van Genderen et al. 1991). The nebula associated with the central object, firstly reported by Hutsemékers \& Van Drom (1991), has a bipolar and filamentary structure, as evidenced by the coronographic imaging performed by Clampin et al. (1995), and subsequently confirmed by Weis et al. (1997) and Nota et al. (1997), who pointed out the similarity to the $\eta$ Car nebula. In particular, Nota et al. (1997) have suggested that the structure of the HR Car nebula emerges from two bipolar lobes, expanding with a maximum velocity of about $115 \mathrm{~km} \mathrm{~s}^{-1}$. The mid-IR images obtained by Voors et al. (1997) reveal that, at arcsec scales, the geometry of the inner nebula around HR Car is not point symmetric with respect to the central star and shows a morphological mismatch with the large-scale structure and the ionized-gas distribution mapped at $12.8 \mu \mathrm{m}$ in the narrow-band [NeII] filter. The authors concluded that the nebula is composed of multiple shells resulting from different mass-loss episodes, probably occurred with a time-dependent geometry. The same conclusion was reached by White (2000) based on radio observations, which indicated a strongly asymmetric inner nebula and an extended emission, whose morphology closely resembles that of the $\mathrm{H} \alpha$ bipolar nebula. The author also suggested the hypothesis that the presence of a companion might account for the asymmetry in the nebula. On the basis of interferometric measurements performed with the Very Large Telescope Interferometer (VLTI) at different epochs, Boffin et al. (2016) have shown that HR Car is a binary system. The authors derived an approximate orbital period between a few to several tens of years, but they ruled out that the detected companion can be the object suggested by White (2000). A detailed mid-IR spectroscopic investigation was performed by Umana et al. (2009), who reported for the first time the existence of photodissociation regions (PDR) in the nebulae surrounding LBV objects and observed an abundance enhancement of $\mathrm{Fe}$ in the gas phase, which is considered as indirect evidence of shocks occurring in the nebula. The presence of amorphous sili-
Table 1. VLT/VISIR Observations Log

\begin{tabular}{cccccc}
\hline Filter & $\begin{array}{c}\lambda_{c} \\
(\mu \mathrm{m})\end{array}$ & Date & $\begin{array}{c}\text { Integration } \\
\text { time }(\mathrm{s})\end{array}$ & Airmass & $\begin{array}{c}\text { FWHM } \\
(\operatorname{arcsec})\end{array}$ \\
\hline SIV & 10.49 & 2011 Feb 23 & 1200 & 1.35 & 0.43 \\
PAH2_2 & 11.88 & 2010 Dec 14 & 2000 & 1.37 & 0.46 \\
\hline
\end{tabular}

cates in the dusty circumstellar medium, together with the lack of crystalline silicates in the inner part of the nebula, suggests that recent dust formation occurred in HR Car during the LBV eruptions.

In this paper, we present the results of a multi-wavelength study of the complex circumstellar environment associated with the LBV HR Car. The analysis is based on a new set of mid-IR, far-IR, and radio images obtained with the Herschel Space Observatory, the Very Large Telescope (VLT), and the Australia Telescope Compact Array (ATCA). In Section 2, we describe the observations and data reduction. In Sect. 3 and 4, we discuss the analysis of the images obtained from each data set, probing different components of the nebula. In Sect. 5, we compare the brightness distribution and the asymmetries at the various wavelengths, with the aim to obtain a comprehensive overview of the envelope and thus explain the observed characteristics. Finally, a summary of the main conclusions is given in Sect. 6

\section{THE DATA SETS}

\subsection{Herschel/PACS imaging}

HR Car was observed as part of both the Hi-GAL (Molinari et al. 2010) key project on 2012 November 14 and the Mass loss of Evolved StarS (MESS) key project (Groenewegen et al. 2011) on 2010 August 16, using the Photodetector Array Camera and Spectrometer (PACS, Poglitsch 2010) on board of the Herschel Space Observatory. The observations were performed by using different observing modes because of the different scientific rationales. HiGAL maps were obtained with the 'fast scan parallel' mode and the final images were generated with pixel sizes of 3.2 and 4.5 arc$\mathrm{sec}$ at 70 and $160 \mu \mathrm{m}$ respectively (Elia et al. 2013). The Hi-GAL pipeline described in Traficante et al. (2011) was used for the data reduction.

PACS MESS observations were performed using the 'scanmap' mode at 70, 100 and $160 \mu \mathrm{m}$. As described by Groenewegen et al. (2011), the images were oversampled by a factor of 3.2 and characterized by pixel sizes of 1 arcsec at 70 and $100 \mu \mathrm{m}$ and $2 \operatorname{arcsec}$ at $160 \mu \mathrm{m}$. More details about the data reduction can be found in Ottensamer et al. (2011). Data analysis was performed on the 'Level 2' data products retrieved from the Herschel Science Archive and no additional data reduction procedure was performed.

\subsection{VLT/VISIR}

Mid-infrared imaging observations of HR Car were conducted in service mode using the VLT Imager and Spectrometer for the mid InfraRed (VISIR, Lagage et al. 2004), mounted on the Cassegrain focus of the VLT Unit Telescope 3 (Melipal). The imaging was carried out through the filters SIV $(10.49 / 0.16 \mu \mathrm{m})$ and PAH2 $(11.88 / 0.37 \mu \mathrm{m})$ on 2011 February 23 and 2010 December 14 respectively. The 0.127 arcsec pixel scale was used, corresponding to 


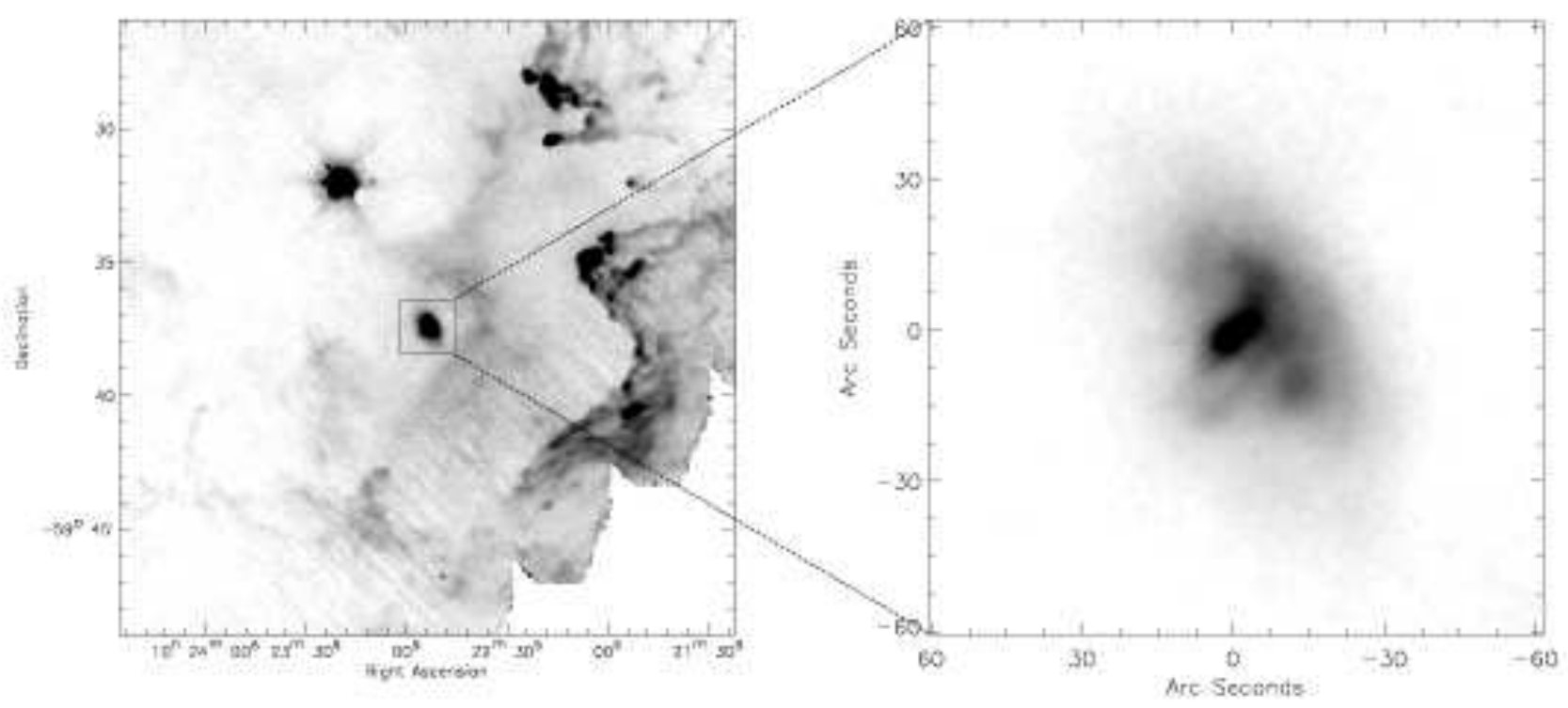

Figure 1. The image of the environment around HR Car, provided by Hi-GAL at $70 \mu \mathrm{m}$, along with the more detailed view of the circumstellar environment from MESS at the same wavelength.

Table 2. Log of the ATCA archive observations

\begin{tabular}{llll}
\hline Project Name & Date & $\begin{array}{l}\text { Frequency } \\
(\mathrm{GHz})\end{array}$ & Array \\
\hline $\mathrm{C} 312$ & 1994 Aug 22/23 & 8.6 & $6 \mathrm{~A}$ \\
$\mathrm{C} 186$ & 1994 Sept 04/05 & 8.6 & $6 \mathrm{~A}$ \\
$\mathrm{C} 312$ & 1995 Jan 02 & 9.2 & $6 \mathrm{~A}$ \\
$\mathrm{C} 312$ & 1995 Apr 11 & $8.3,9.0$ & $6 \mathrm{C}$ \\
$\mathrm{C} 186$ & 1995 Nov 08/09 & 8.6 & $6 \mathrm{~A}$ \\
$\mathrm{C} 1167$ & 2004 July 15 & 18 & $6 \mathrm{~A}$ \\
$\mathrm{C} 2943$ & 2014 Dec 24 & $5.4,9.0,44.0$ & $6 \mathrm{~A}$ \\
\hline
\end{tabular}

a 32 ." $5 \times 32$ ". 5 field of view. The standard chopping/nodding technique was applied to remove the sky contribution, with chopping and nodding throw angles of 20 arcsec and a chopping frequency of $0.25 \mathrm{~Hz}$. To further improve the image quality, a random jitter pattern with a maximum throw of 3 arcsec was superimposed on the nodding sequence. The standard stars HD89682 and HD152880 were observed and used for photometric calibration in both bands. Such observations were also used to determine the FWHM of standard star images and thus to derive the actual angular resolution of our final maps. A summary of the VISIR observations is presented in Table 1, including the date of the observations, the exposure time, the airmass at the beginning of the observations, and the FWHM.

The images were processed using the standard pipeline for data reduction provided by ESO (version 3.3.1). The chopped and nodded images were then combined to make a single image for each filter.

\subsection{ATCA}

We observed HR Car with the ATCA in 2014, on 24 December, with the array in $6 \mathrm{~A}$ configuration, using the Compact Ar- ray Broadband Backend ( $\mathrm{CABB}$ ), which provides two independent intermediate-frequency (IF) outputs of $2 \mathrm{GHz}$ in dual linear polarization. The data were taken interleaving simultaneous observations at two frequency pairs: $5.5 / 9 \mathrm{GHz}$ and $43 / 45 \mathrm{GHz}$. All the observations used the $1 \mathrm{M}$ mode, which samples $20481-\mathrm{MHz}$ wide channels.

Bandpass and flux calibration were performed with observations of the standard calibrator 1934-638. For the phase calibration we used 1045-62. The beam size of the observations was $11^{\prime \prime} 9 \times 1{ }^{\prime \prime} 5$, $1^{\prime \prime} 3 \times 1$." 1 , and $0 . " 7 \times 0$." 2 for the $5.5,9$, and $44 \mathrm{GHz}$ observations, respectively.

We used the MIRIAD data reduction package to process all data sets. We split the CABB $4 \mathrm{~cm}$ band in two 2-GHz sub-bands, one centred at $5.5 \mathrm{GHz}$ and the other at $9 \mathrm{GHz}$, then we calibrated and imaged the data across the entire 2-GHz bands. For the data set obtained with the $7 \mathrm{~mm}$ receiver, we merged the two bands centred at 43 and $45 \mathrm{GHz}$ after the calibration process to obtain an average map with a high signal-to-noise ratio. In all these cases, the broad-band data were weighted with the robust parameter setted at 0.5 and multi-frequency synthesis was applied, using the MIRIAD task INVERT. The images were subsequently deconvolved with the CLEAN algorithm, as implemented in the task MFCLEAN, and finally restored with a synthesised beam (task RESTOR).

\subsubsection{Archival Data}

The Australia Telescope Online Archive (ATOA) was queried for observations of HR Car. In addition to project C312 and C186 (White 2000; Duncan \& White 2002), project C1167 targeted HR Car at $18 \mathrm{GHz}$. We selected the observations performed with $6 \mathrm{~km}$ array configurations, to obtain homogeneous data sets for map comparison. Table 2 summarises the dates and observing frequencies of these observations. For a better comparison with our data set, these observations were processed following the same scheme 


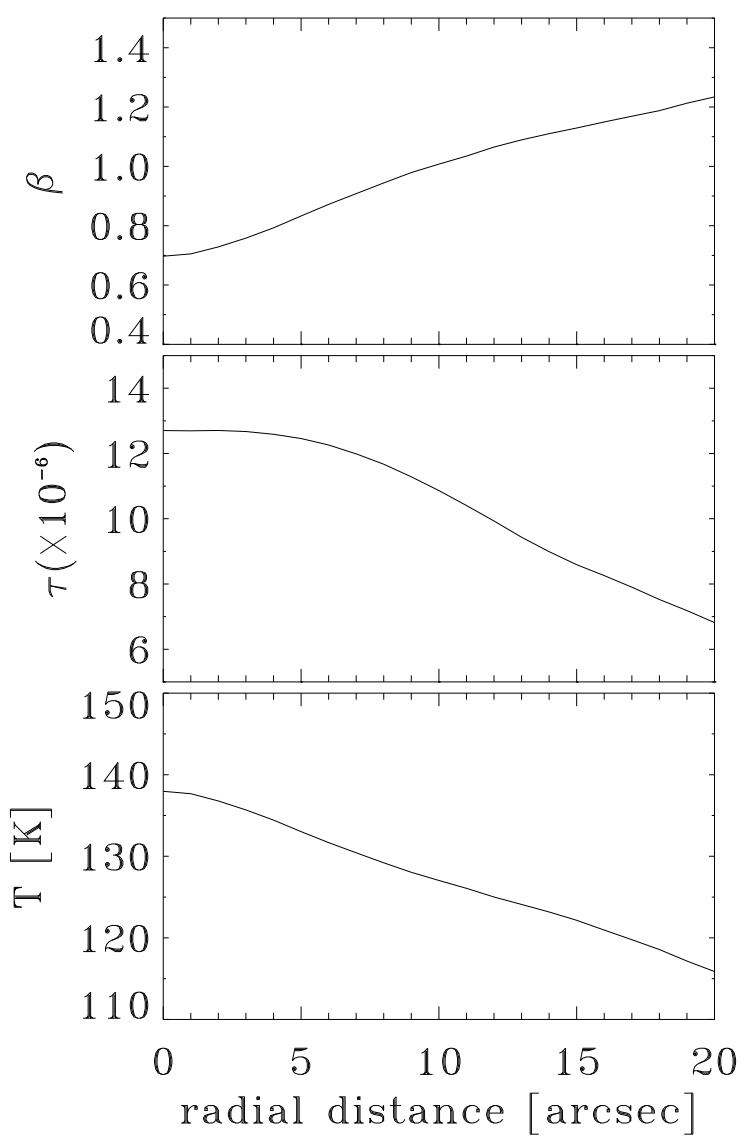

Figure 2. Average radial profiles of the dust temperature (T), opacity index $(\beta)$, and optical depth $\left(\tau_{0}\right)$ maps, as obtained by a pixel to pixel fit of the SED by a modified single-temperature blackbody (see text).

described in the previous section. All the observations performed at the same frequency were concatenated in a stacked data set to produce a single map. The achieved spatial resolution (FWHM) and rms noise level are $1.2 \times 1.2 \operatorname{arcsec}^{2}$ and $2.4 \times 10^{-5} \mathrm{Jy} \mathrm{beam}^{-1}$ at

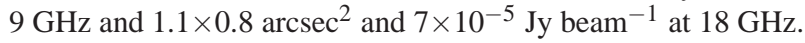

\section{SHAPE AND STRUCTURE OF THE DUSTY NEBULA}

\subsection{The extended nebula}

In Figure 1, we present the complex environment around HR Car as found in the $70 \mu \mathrm{m}$ Hi-GAL PACS image. It shows extended dusty clouds and very clear complexes of fingers of emission in the West side. The outermost and brightest structures are probably associated with HD 302821, as the pillars point toward the supergiant located about 6 arcmin away from HR Car in the NE direction. On the other hand, the fainter arc-shaped emission at the western side of the HR Car nebula is located at the same distance of HR Car and is probably interstellar material that has been swept up by the stellar wind (Nota et al. 1997).

Together with the higher-resolution 70- and 100- $\mu$ m images from MESS, these maps provide us with valuable information on the structure of the dusty nebula, revealing a circumstellar emission much more extended and complex than previously known. The presence of a cold dust component in the circumstellar environment
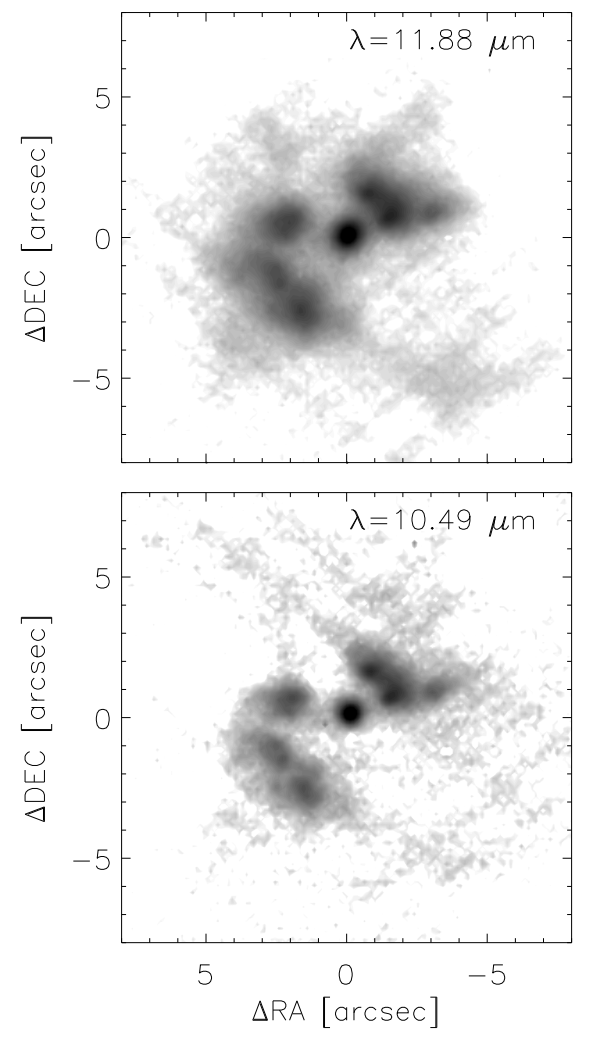

Figure 3. HR Car in the PAH2_2 and SIV filters with VISIR camera. North is up, and East points to the left.

of HR Car was suggested by Lamers et al. (1996), to account for the jump observed in the spectra taken in different bands with the ISO-Short Wavelength Spectrometer.

The nebula has an essentially elliptical shape, with the major axis oriented almost along the NE-SW direction. Its size is about $50^{\prime \prime} \times 70^{\prime \prime}$, that is about $1 \times 1.5 \mathrm{pc}$ at $5 \mathrm{kpc}$ (P.A. $\sim 30^{\circ}$ North to East) and has an integrated flux density at $70 \mu \mathrm{m}$ of about $23.65 \mathrm{Jy}$.

The most noticeable characteristic is the distribution of the emitting material, which extends mainly to the NW side with respect to the central star, suggesting a high degree of asymmetry in the material lost by the star. The comparison of the Herschel image with the $\mathrm{H} \alpha$ one (Fig. 1 in Nota et al. 1997) shows that the brightest part of the infrared emission correlates with the $\mathrm{H} \alpha$ features, resembling the bipolar lobes lying in the SENW direction. The $\mathrm{H} \alpha$ Southern lobe is also clearly noticeable, but it is evident that the dusty envelope extends well beyond the ionized-gas component, especially to the North-East side of the nebula. Driven by the structured dust-envelope appearance, we focused on the analysis of the Spectral Energy Distribution (SED) across the nebula, with the aim of deriving some hints of possible variations in the dust characteristics. For each pixel of the 70- $\mu$ m map, a SED was thus constructed using the MESS 70-, 100-, and 160- $\mu \mathrm{m}$ maps and the 12- and 22- $\mu \mathrm{m}$ from Wide-field Infrared Survey Explorer (WISE; Wright et al. 2010) images. All the maps were convolved with the same beam of 16.8 arcsec (that is the FWHM of the WISE $22-\mu \mathrm{m}$ co-added images) and then regridded to correspond the pixel-size with the $70-\mu \mathrm{m}$ images. The SED of each pixel was then fitted by a single-temperature blackbody modified by an opacity index $(\beta)$. 


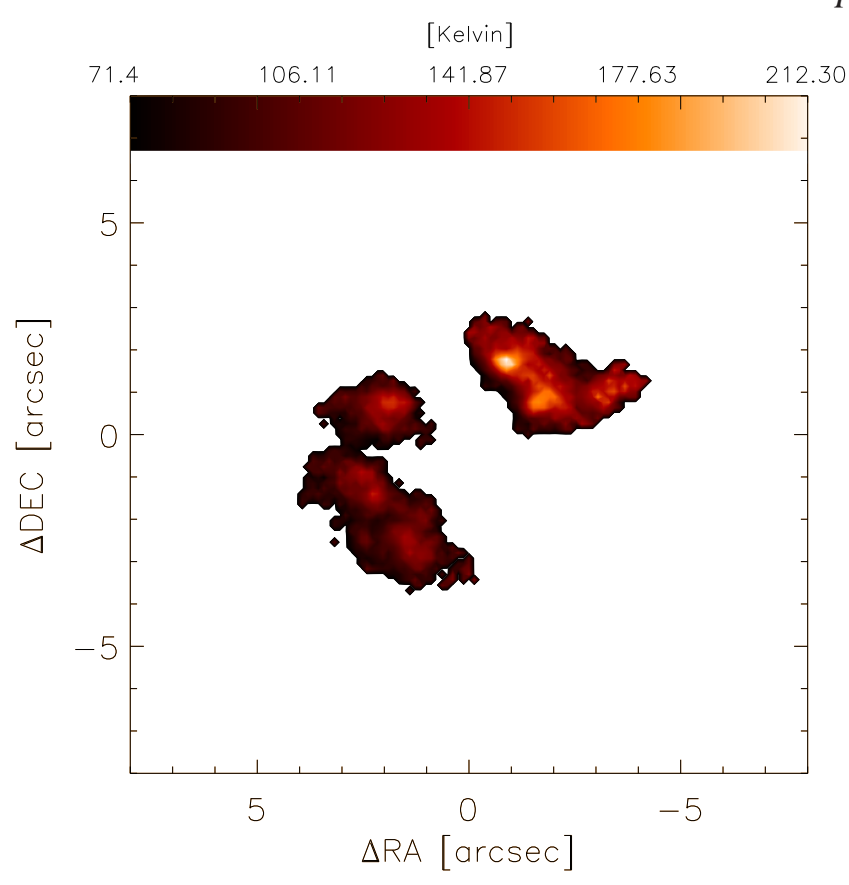

Figure 4. Temperature (colour) map derived by combining the images at 10.49 and $11.88 \mu \mathrm{m}$.

Assuming that the flux density $F_{v}$ at frequency $v$ is given by

$$
F_{V} \propto B_{V}(T) \times\left(1-e^{-\tau_{v}}\right), \text { where } \tau_{v}=\tau_{0} \times\left(v / v_{0}\right)^{\beta}
$$

where $B_{v}$ and $\tau_{v}$ are the Planck intensity and the optical depth at frequency $v$, from the fit we obtained the dust temperature $T$, the opacity $\beta$ and the optical depth $\tau_{0}$ at the chosen reference wavelength $\left(c / v_{0}=100 \mu \mathrm{m}\right)$. The IDL code MPFITFUN based on a least-square $\chi^{2}$ fit has been used. In Figure 2 the average radial profiles for the resulting $T, \beta$, and $\tau_{0}$ maps starting from the central star position are shown. The profiles suggest the presence of a hot dust region at distance smaller than about 8 arcsec from the central object, where the optical depth is higher, and of a more extended colder and optically thinner halo. Also the dust opacity shows a radial gradient, with increasing values of $\beta$ as we move away from the central core. Values of $\beta$ close to 2 at far-IR wavelengths are expected for standard small grains characterising the ISM, while smaller values of $\beta$ are usually attributed to larger grains, resulting from grain accretion. The radial dependence may indicate a change in the properties of the circumstellar dust, with more processed dust located in the inner region, where the eruptive episodes create the conditions favourable to the growth of dust grains (Kochanek 2011), and smaller or more fragmented grains far from the star, where it is possible that the ejected material is mixed with preexisting ISM.

The approximate dust mass of the emitting envelope can be derived from the optical depth map, by dividing it for the absorption coefficient per mass unit and then by integrating over all the pixels with brightness higher than $3 \sigma$ (Umana et al. 2010). The absorption coefficient for mass unity was computed using the package OPACITYTOOL given by the DIANA project ${ }^{1}$. Assuming standard astronomical silicates, as suggested by the silicate emission bump observed by Umana et al. (2009), we derive

\footnotetext{
${ }^{1}$ http://www.diana-project.com/data-results-downloads/fortran-package-
} to-compute-diana-standard-dust-opacities/
Table 3. Properties of the new radio maps.

\begin{tabular}{cccccc}
\hline Band & $\begin{array}{c}\text { Frequency } \\
{[\mathrm{GHz}]}\end{array}$ & $\begin{array}{c}\text { HPBW } \\
{[\operatorname{arcsec}]}\end{array}$ & $\begin{array}{c}\text { LAS } \\
{[\operatorname{arcsec}]}\end{array}$ & $\begin{array}{c}\text { Flux density } \\
{[\mathrm{mJy}]}\end{array}$ & $\begin{array}{c}\text { rms } \\
{\left[\mathrm{mJy} \mathrm{beam}^{-1}\right]}\end{array}$ \\
\hline $\mathrm{C}$ & 5.4 & $1.9 \times 1.5$ & 20.4 & 15.28 & 0.02 \\
$\mathrm{X}$ & 9.0 & $1.3 \times 1.1$ & 12.2 & 13.87 & 0.03 \\
$\mathrm{Q}$ & 44.0 & $0.7 \times 0.2$ & 2.5 & 5.9 & 0.2 \\
\hline
\end{tabular}

a $\kappa_{100}=66.4 \mathrm{~cm}^{-2} \mathrm{~g}^{-1}$ and a dust mass, within 70 arcsec, of $M_{d}=1.6 \times 10^{-3} \mathrm{M}_{\odot}$ (assuming a distance of $5.4 \mathrm{kpc}$ ). As the infrared observations just trace the hotter dust emission, such value has to be considered as a lower limit to the entire envelope dust mass content. Assuming a dust-to-gas ratio of 200 for the circumstellar material, we can estimate a total nebular mass of at least $0.3 \mathrm{M}_{\odot}$. This result is close to the estimate of $0.15 \mathrm{M}_{\odot}$ obtained by Hutsemékers \& Van Drom (1991) assuming a bipolar cone geometry, and is consistent with the estimates of $0.8 \mathrm{M}_{\odot}$ and $2.1 \mathrm{M}_{\odot}$ derived by White (2000) and Clampin et al. (1995). Note, however, that in some cases the mass estimates could be affected by the uncertainty on the nebular geometry, which in HR Car seems to be very complex.

\subsection{The inner dust envelope}

Through the mid-IR images of HR Car (Fig. 3), it is possible to investigate more deeply the inner dusty circumstellar envelope (CSE) and obtain valuable information about it. HR Car was mapped by Voors et al. (1997) in the mid-IR $N$ filter with the instrument TIMMI (Thermal Infrared Multi Mode Instrument). They reported about a quite compact dusty nebula, not symmetrically distributed around the central star. The better resolution and the high sensitivity of the VLT allow us to discern finer details of the nebular morphology. The two maps at 10.49 and $11.88 \mu \mathrm{m}$ exhibit very similar features, with the emission mainly located along two arcs of different intensity, extending at least $\sim 5$ arcsec South-East and 4 arcsec North-West of the central source. In addition, the lowest flux level in the PAH2_2 map reveals a very faint extended halo around the bright structures, visible out to 7 arcsec from the centre, which is not detected in the SIV map. Given the large extension of the dusty nebula revealed by the PACS images, it is important to note that if the cloud were more extended than the chop positions, then any large-scale emission - if present - would be cancelled out in the mid-IR observations.

Under the assumption that the dust emission is optically thin at these wavelengths, by comparing the two images in the two bands, we derived the colour-temperature map displayed in Fig. 4, as described in (Umana et al. 2010). The map shows a temperature gradient across the CSE and the resulting colour temperatures are in very good agreement with those obtained from the MESS maps. The higher spatial resolution of the VISIR images indicates that the NW arc is cooler than the SE crescent.

The optical-depth map at $10.49 \mu \mathrm{m}$ was calculated from the relation $I_{v} \approx B_{v}(T) \tau_{v}$. The resulting dust mass (obtained as explained in the previous section) is $M_{d}=2.3 \times 10^{-4} \mathrm{M}_{\odot}$. This indicates that the mass of the hotter dust lying within $5^{\prime \prime}$ is about 14 percent of the mass of the entire dust envelope emitting in the far-IR. 

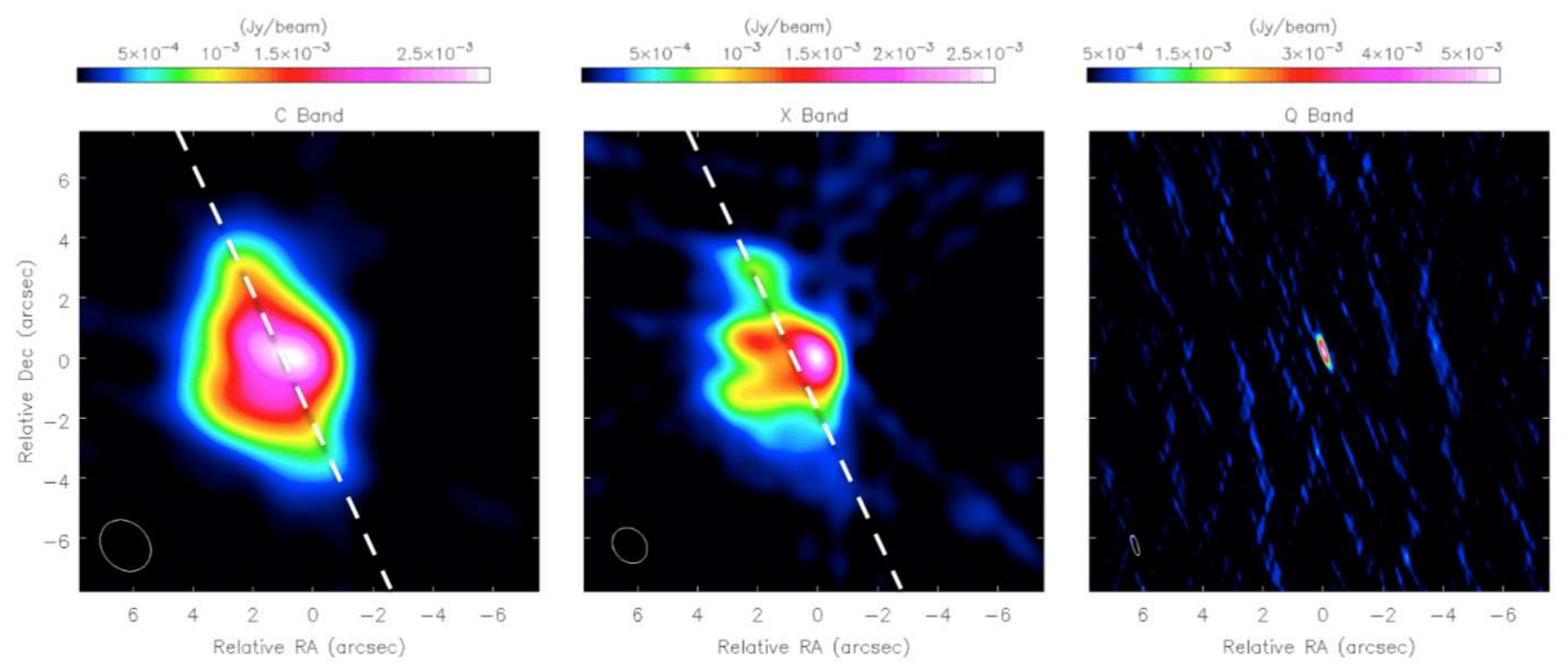

Figure 5. The new ATCA radio images of HR Car at 5 (left), 9 (centre), and 44 (right) GHz. The dashed line indicates the major axis of the nebular elongation and is inclined of $25^{\circ}$ with respect to the North-South direction.

\section{RADIO EMISSION}

Final images for radio bands at 5.5, 9, and $44 \mathrm{GHz}$ are presented in Figure 5. Table 3.2 reports the map properties and the measured fluxes, together with the array largest angular scale (LAS). The emission at 5.5 and $9 \mathrm{GHz}$ traces in detail the structure of the extended ionized circumstellar nebula, which appears elongated $\approx 25^{\circ}$ in the North to East direction. The resolution of the $44 \mathrm{GHz}$ map allowed us to separate the stellar radio source from the extended ionized circumstellar nebula, filtered out at this frequency because of the small LAS. The compact source is at $\alpha=$ $10^{\mathrm{h}} 22^{\mathrm{m}} 53.9, \delta=-59^{\circ} 37^{\prime} 28^{\prime \prime} \cdot 3$ [J2000] and its flux density is equal to $F(44 \mathrm{GHz})=5.9 \pm 0.2 \mathrm{mJy}$.

Consistently with previous radio observations, the 5 and 9$\mathrm{GHz}$ images reveal the morphology and the internal structure of the inner nebular core, showing a clear eastward displacement of the ionized region with respect to the peak of the emission, located at the position of the compact source observed at $44 \mathrm{GHz}$. The comparison of our maps with those obtained by Duncan \& White (2002) points to some differences in the emitting features, despite the similarity in angular size and measured fluxes and the elongated elliptical morphology. In Figure 6, the 9-GHz map is displayed along with the 9 and $18-\mathrm{GHz}$ maps obtained from the ATCA archive data sets. For a better comparison, we convolved and regridded all the maps to a common pixel size of 0.17 arcsec and resolution of $1.27 \times 1.07 \operatorname{arcsec}^{2}$. First of all, we notice that the 1995 map exhibits a main component coincident with the stellar object and a second clump at the eastern side of it, having a quite similar flux level. In our images, the bright emission at the central star position clearly outshines the eastward feature, which shows a horseshoe shape extending along two arms oriented roughly horizontally, more evident in the $9-\mathrm{GHz}$ image.

With the aim of investigating the mechanisms responsible for the radio emission, the observations at 5 and $9 \mathrm{GHz}$ were used to generate the map of the radio spectral index $\alpha$, defined as $F_{V} \propto$ $v^{\alpha}$. The images were obtained with the same parameters of the reduction tasks as for our own data. In order to reach the same angular resolution at the two frequencies, we restored both maps with Gaussian beams of the same sizes. Pixels whose values were below $5 \sigma$ were discarded.

In Figure 7, we present the spectral-index map and the contour of the same map superimposed on the VISIR PAH2_2 image. It can be seen that the spectral-index distribution is not uniform across the emitting region, indicating the presence of different components. At the stellar position, the spectral index is $\alpha=0.55$, indicating that in this region the radio emission is dominated by stellar-wind emission. In the remaining part of the nebula, the spectral index ranges from values close to -0.1 , typical of a free-free emission, to steeper values between -0.4 and -0.7 toward the edges of the radio-emitting region. Thus, though most of the diffuse emission is compatible with thermal emission, there are hints of a non-thermal emission component at the boundary of the brightest SE arm of the mid-IR emitting nebula (Fig. 7).

On the basis of the spectral-index map analysis, we assume that the flux measured at $44 \mathrm{GHz}$ is due to thermal emission from the stellar wind. Therefore, the current mass-loss rate can be estimated from the relation derived by Panagia \& Felli (1975):

$$
\dot{M}=6.7 \times 10^{-4} v_{\infty} F_{v}^{3 / 4} D^{3 / 2}\left(v \times g_{f f}\right)^{-1 / 2}\left[M_{\odot} y r^{-1}\right],
$$

where $v_{\infty}=150 \mathrm{~km} \mathrm{~s}^{-1}$ is the terminal velocity of the wind (Hutsemékers \& Van Drom 1991), $D=5.4 \mathrm{kpc}$ is the source distance (van Genderen et al. 1991) and $g_{f f}$ is the Gaunt factor, approximated with $g_{f f}=9.77\left(1+0.13 \log \frac{T^{3 / 2}}{v}\right.$ ) (Leitherer \& Robert 1991). Assuming a temperature of the plasma in the wind of $10^{4} \mathrm{~K}$, we find that in 2014 the star was losing mass at a rate of $1.17 \times 10^{-5} \mathrm{M}_{\odot} \mathrm{yr}^{-1}$. The comparison between the new image at $9 \mathrm{GHz}$ and earlier epoch maps (Fig. 6) highlights a flux density enhancement at the central star position. White (2000) estimated, in fact, a total flux of $1.0 \mathrm{mJy}$ at $9 \mathrm{GHz}$ for the compact stellar component, against the $2.3 \mathrm{mJy}$ derived by scaling the flux mea- 

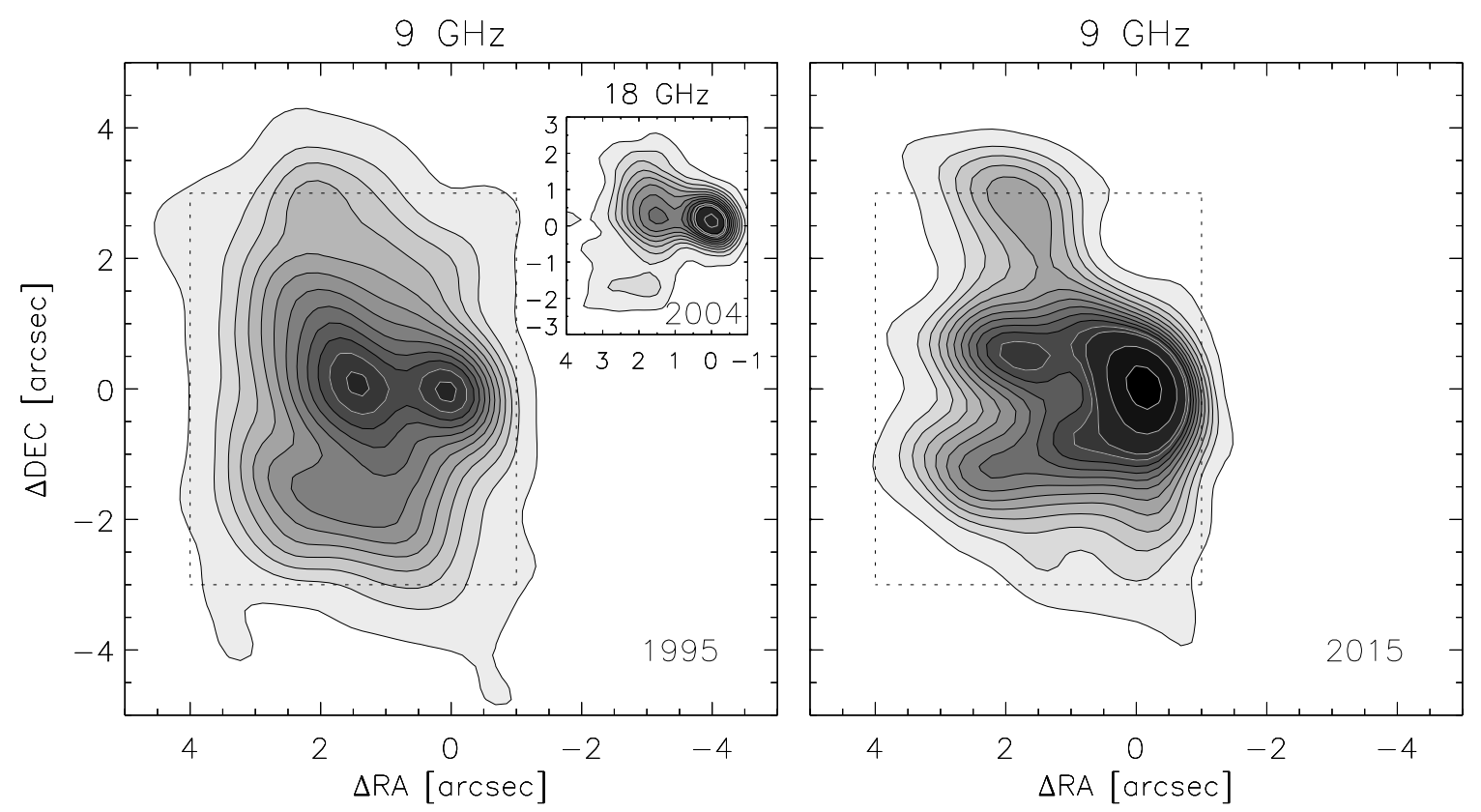

Figure 6. VLA $9 \mathrm{GHz}$ and $18 \mathrm{GHz}$ images at different epochs. The contours are drawn at the levels $1.5,3,4,5,6,7,8,9,10,11,12,13,17$, and 23 of the rms in the maps, that is $1 \times 10^{-4} \mathrm{mJy}_{\text {beam }}{ }^{-1}$. For all the images, the half power beam width (HPBW) of the synthesised beam is $1.27 \times 1.07 \mathrm{arcsec}^{2}$ and the pixel scales are set to $0.17 \mathrm{arcsec}_{\text {pixel }}{ }^{-1}$

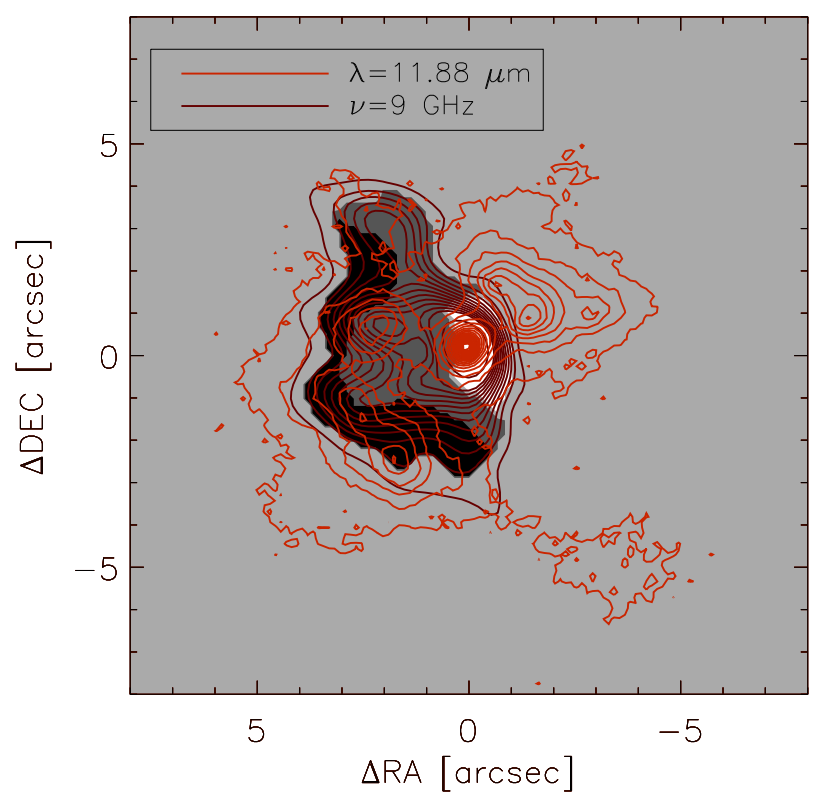

Figure 7. Two frequency $(5.5-9 \mathrm{GHz})$ spectral index intensity map with superimposed the 9-GHz (left panel) and the PAH2_2 (right contour) map contours. The black area represents the region with $\alpha \leqslant-0.4$ (average -0.6 ), the grey area the region with $-0.4 \leqslant \alpha \leqslant+0.2$ (average -0.15 ) and the white area the region with $\alpha \geqslant+0.2$ (average value +0.45 ).

sured in 2014 at $44 \mathrm{GHz}$ according to $\mathrm{F}_{V} \propto v^{0.6}$. The mass loss rate derived by assuming $v=9 \mathrm{GHz}$ and $\mathrm{F}_{v}=1 \mathrm{mJy}$ in Eq. 1 is $6.1 \times 10^{-6} \mathrm{M}_{\odot} \mathrm{yr}^{-1}$ between 1994 and 1995, that corresponds to a mass loss enhancement by a factor of $\sim 1.9$.
HR Car is known to be characterised by photometric variability, as shown by the data in the AAVSO website, and has brightened by almost two visual magnitudes over the past decade. During 1994 and 1995 the source was in a phase of visual minimum of the S-Doradus cycle before it rose to the maximum, which began in 1996. In 2014 the star was again in the minimum phase, which is still on-going after the maximum ended in 2006. The visual magnitude at the minimum of 1995-1996 was 0.3-0.4 lower than in 2014, contrary to the lower mass-loss rate estimated. Such difference could be interpreted in terms of the bistability mechanism of line-driven winds, as a consequence of a change in the iron ionization structure (Vink \& de Koter 2002), as it was hypothesised in the case of AG Car (Groh et al. 1999) and Iras 18576-0341 (Buemi et al. 2010). On the other hand, the different mass-loss rate measured during the two minima could be related to the binariety of HR Car, recently confirmed by Boffin et al. (2016), who constrained the time of the periastron passage between 2013.2 and 2015.05, and most likely in late 2014. The authors could not fully constrain the orbital period, but their best-fitting value corresponds to 12 years. This orbit parameters, if confirmed, locate the two stars much closer in 2014 than in 1994-1995, suggesting possible interactions between the stellar components that could affect the massloss rate near the periastron epoch.

To complement the study of flux variability, the ATCA archive was queried for observations with similar angular resolution, but no additional data were found on the time-line between the two data sets, with the exception of the $18 \mathrm{GHz}$ observations performed in 2004 July, not previously published. The resulting map (Fig. 6) shows a doubly-peaked structure similar to the 1995 image, in excellent agreement with the flux from White (2000) under the assumption of spherical stellar-wind emission. Together with the similarity in the structure of the nebula, this indicates that the measured flux variation took place after 2004, but the large temporal gap be- 

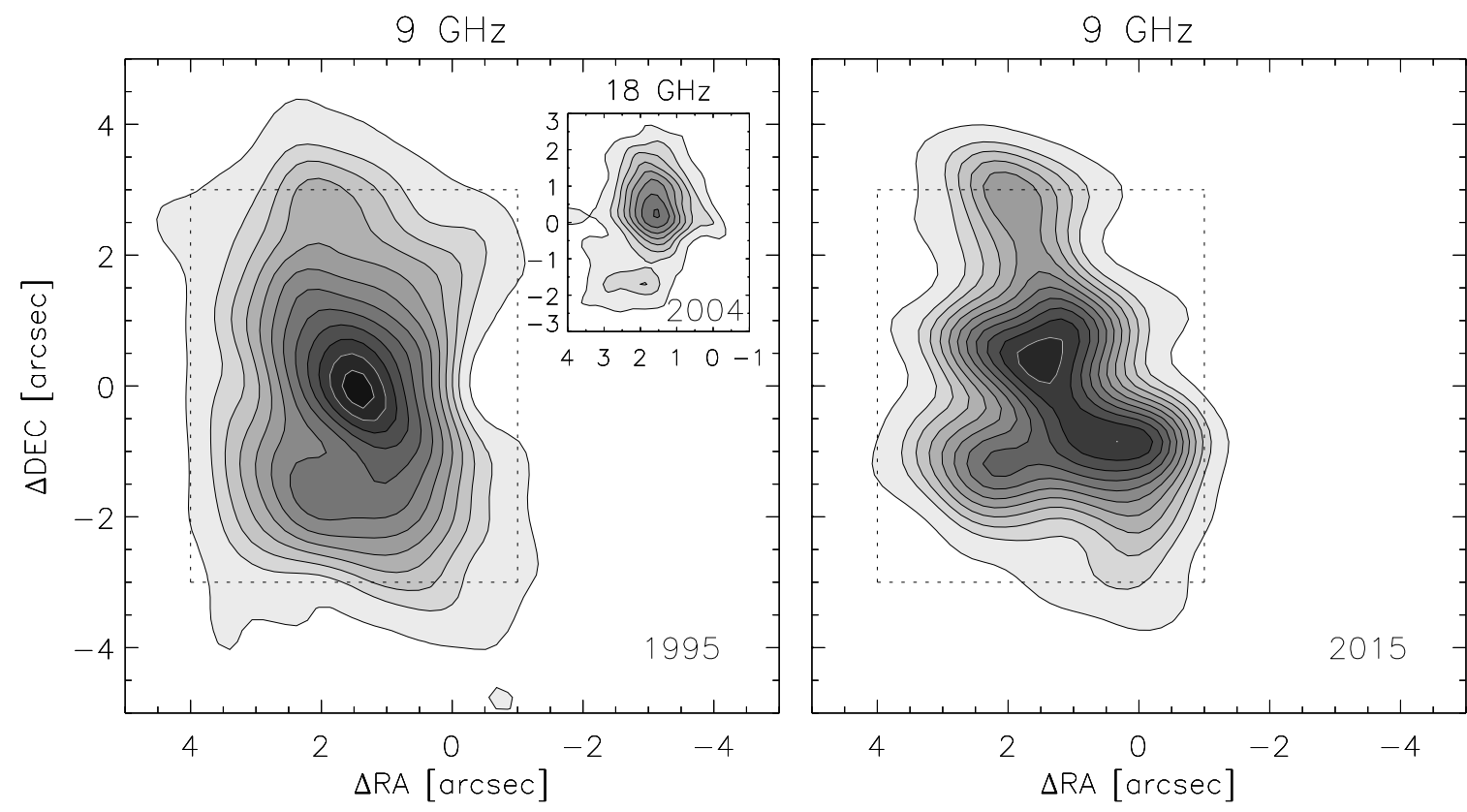

Figure 8. The same maps as in Fig. 6, after the subtraction of the stellar component (see text).

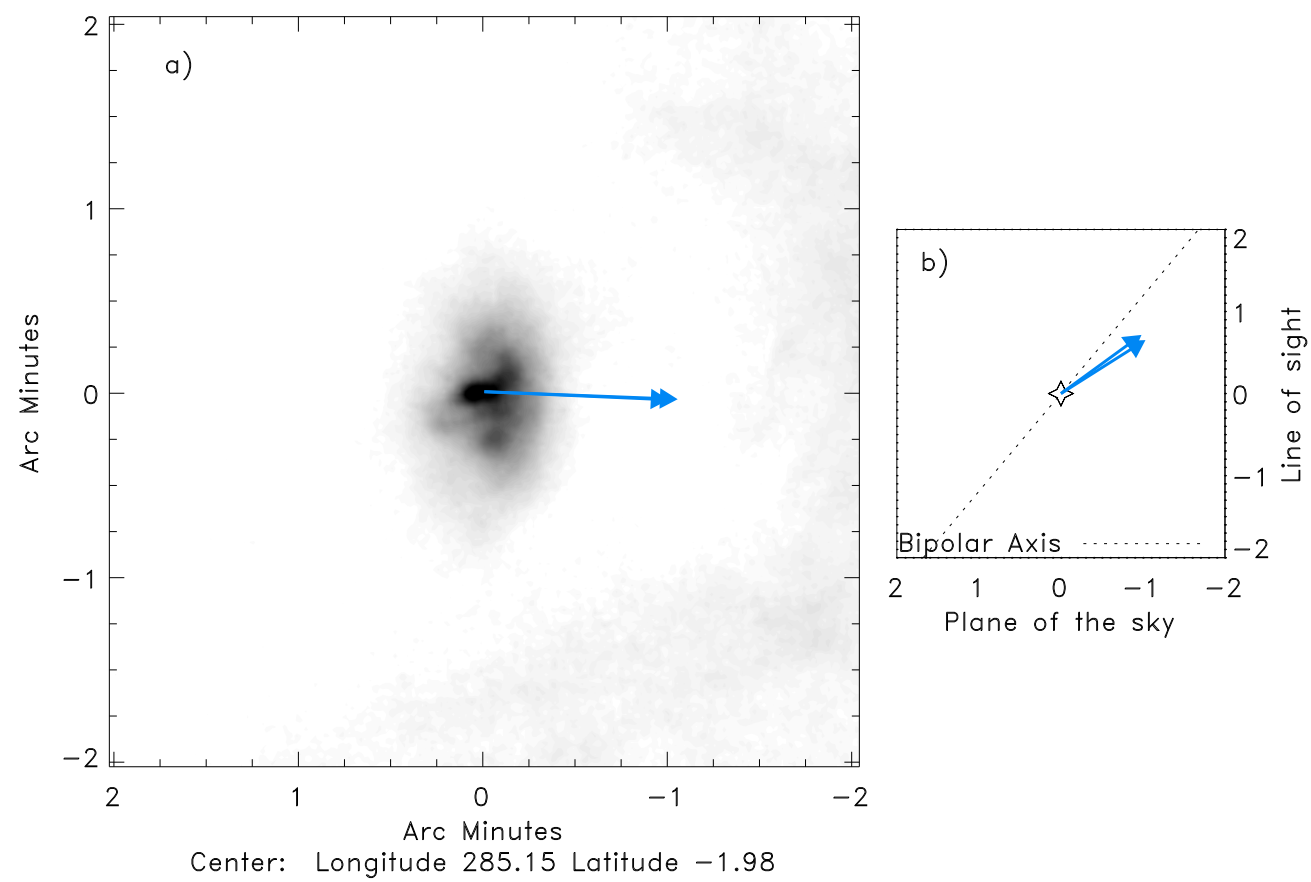

Figure 9. Panel a) shows the PACS image of HR Car, where the arrows indicate the direction of the space motion corrected for the solar motion. In panel b) the same arrows are sketched in a top view $\left(90^{\circ}\right.$ out of the plane of the sky), to emphasise the angle between the direction of the space motion and the bipolar axis (dashed line), which is about $16^{\circ}$.

tween the observations makes it impossible to rule out that further changes occurred.

To separate the nebular and the stellar-wind emission, a point source component at the stellar position has been subtracted from the data sets in the Fourier plane, via the MIRIAD task UVMODEL, where the assumed model flux density was 1.0 and $2.3 \mathrm{mJy}$ for the
Duncan \& White (2002) data set and ours, respectively. The maps of the residuals (Fig. 8) show that some differences are also present in the radio nebula. In particular, the main differences are in the inner part of the nebula, where the emission is arc-shaped. This morphology may derive from a different density distribution (e.g. 
from new mass injection) or from the shielding of the stellar ionizing radiation.

\section{DISCUSSION}

Because of the different morphology of the nebula unveiled by the observations at various wavelengths, determining the actual structure of the circumstellar environment that surrounds HR Car is a tricky task: it appears to be complex and probably the result of different mass-loss episodes. The great asymmetry in the large-scale structure of the nebula revealed by the far-IR images can be explained in terms of density variation in the cloud. The asymmetry might be the result of stellar evolution in a circumstellar and/or interstellar medium characterised by a radially decreasing density. However, it is difficult to assess if such inhomogeneity is preexisting in the ISM around HR Car or is a consequence of its massloss process. We also explored the possibility that the arc-shaped morphology of the extended dusty nebula could have formed by compression in a bow-shock, driven by the stellar wind impacting on the surrounding medium, in the direction of the space motion of HR Car.

Bow shock structures have been observed in the far-IR around different stellar objects, e.g. AGBs, red supergiants and Algols (Ueta et al. 2008; Jorissen et al. 2011; Cox et al. 2012; Mayer et al. 2016). First of all, we checked if the velocity direction of HR Car is compatible with the location of the bow shock. The Galactic velocity of HR Car was determined following the calculation of Johnson \& Soderblom (1987). We assumed a proper motion of $\mu_{\alpha}=-6.87 \mathrm{mas} / \mathrm{yr}$ and $\mu_{\delta}=2.33 \mathrm{mas} / \mathrm{yr}$ (van Leeuwen 2007) and a distance of $5.4 \mathrm{kpc}$. Since Nota et al. (1997) gave two possible values of heliocentric radial velocity, -14 and -22 $\mathrm{km} \mathrm{sec}-1$, we calculated the Galactic velocity for both cases. Taking into account the solar motion, in the first case we found $[U, V, W]=[-117.9,-29.5,-4.3] \mathrm{km} \mathrm{sec}^{-1}$, while in the second $[U, V, W]=[-120.0,-21.8,-4.0] \mathrm{km} \mathrm{sec}^{-1}$.

In both cases, the velocity vector lies almost perfectly on the Galactic plane with angles of $2.0^{\circ}$ and $1.9^{\circ}$, respectively, which are very close to the direction of the line connecting the stellar position to the apex of the parabolic curve that defines the western boundary of the far-IR emission (Fig. 9). A further issue with such scenario is the comparison of the nebular dimension with the expected wind stand-off distance, defined as the distance $r$ from the star where the momentum flux in the wind equals the ram pressure of the ambient medium. Under the assumption of a sphericallysymmetric and time-invariable stellar wind and of a homogeneous ambient medium, the distance $r_{0}$ between the star and the apex of the bow (stagnation point) can be derived by the ram pressure balance of the wind and ambient medium:

$$
\begin{gathered}
r_{0}=0.18\left(\frac{\dot{M}}{10^{-5} M_{\odot} \mathrm{yr}^{-1}}\right)^{\frac{1}{2}}\left(\frac{v_{w}}{10 \mathrm{~km} \mathrm{~s}^{-1}}\right)^{\frac{1}{2}}\left(\frac{n_{A M}}{\mathrm{~cm}^{-3}}\right)^{-\frac{1}{2}} \times \\
\times\left(\frac{v_{\star}}{100 \mathrm{~km} \mathrm{~s}^{-1}}\right)^{-1} \mathrm{pc},
\end{gathered}
$$

where $v_{w}$ is the wind terminal velocity, $n_{A M}$ is the ambient medium density, and $v_{\star}$ is the velocity of the star (Chiotellis et al. 2016). The ambient density was derived using the relation given by (Mihalas \& Binney 1981), which relates the interstellar hydrogen nucleus density $n_{\mathrm{H}}$ to the distance from the Galactic plane $z$ :

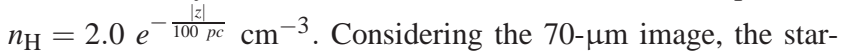
apex distance projected on the sky plane is about $25 \mathrm{arcsec}$, which corresponds to $0.58 \mathrm{pc}$ at $5.4 \mathrm{kpc}$. This distance is equal to $0.76 \mathrm{pc}$ when de-projected following the system inclination with the respect to the plane of the sky proposed by Nota et al. (1997). Adopting $v_{\star}=120 \mathrm{~km} \mathrm{sec}^{-1}$ and $v_{w}=115 \mathrm{~km} \mathrm{sec}^{-1}$ (Nota et al. 1997), from Eq. (2) the stand-off distances of $0.76 \mathrm{pc}$ is compatible with a bow shock caused by a mass loss rate of $6 \times 10^{-6} \mathrm{M}_{\odot} \mathrm{yr}^{-1}$. Despite the rough approximations adopted, this value is in good accordance with the mass-loss rate typical of the LBV phase, as well as with the values derived for HR Car on the basis of the radio data (Sec. 4).

It is noteworthy that the derived stellar velocity vector lies very close to the bipolar axis of the $\mathrm{H} \alpha$ nebula (see panel b of Fig. 9). This suggests that the density enhancement produced in the bow shock may affect the distribution shape of the ejected material.

At shorter wavelengths, the VISIR images reveal a less extended emission and a quite different inner nebular morphology, with brighter emission concentrated mostly in two arc-like structures that can be interpreted as the results of the limb brightening of a circumstellar envelope close to the central star. The radio maps give us a detailed view of the innermost part of the ionized gas component of CSE, whose morphology adds one more piece to the puzzle of this complex environment. The radio images show a marked asymmetry in the emission distribution with respect to the central object, but the asymmetry direction is opposite to that in the far-IR emission.

\subsection{The expanding-lobe model}

Voors et al. (1997) suggested that the CSE around HR Car is the result of multiple outflows with a bipolar geometry. This was also proposed by Nota et al. (1997) on the basis of high-resolution coronographic observations. The author interpreted the morphological and kinematic analysis of the HR Car nebula as a large-scale bipolar structure, very reminiscent of the $\eta$ Car nebula, where the $\mathrm{H} \alpha$ filaments are the signature of two symmetrical expanding bubbles. The author hypothesised that the presence of bipolar lobes may imply an interaction between the fast stellar wind and a preexisting CSE, in the form of an asymmetrical envelope or an equatorial dust density enhancement.

In the framework of the first scenario, a simple geometrical model of the two bubbles originating from the star is sketched in Figure 10. The three-dimensional orientation of the structure is the same of the HR Car H $\alpha$ nebula derived by Nota et al. (1997), with an inclination of the axis of the "homunculus" to the plane of the sky of $50^{\circ}$. Using de-projected radii of 2.5 and 4 arcsec for the outer edges of the two expanding shells and 0.5 arcsec for the shell thickness, the maxima in the optical depth, under the assumption of optically-thin conditions, overlap with the maximum of the midIR intensity maps. The good agreement of the geometric parameters derived by Nota et al. (1997) with those used in our geometric toy model could indicate that there are no significant changes in the orientation of the outflows that create the structures at different epochs.

By a comparison of our mid-IR and radio maps, the inner and bright ionized-gas emission is apparently confined within the dust and the warmer arc (see Fig. 4) seems to trace the western boundary of the radio emission (Fig. 7). This suggests that the dust is optically thick to the UV photons, absorbing a portion of the stellar radiation and thus shielding the circumstellar gas from the ionizing flux. The overall elongation observed in lower-frequency radio maps could indicate the direction in which the radiative flux is channelled by the surrounding medium. Apart from the stellarwind emission, the spectral index appears to be flat $(\approx-0.1)$ across 

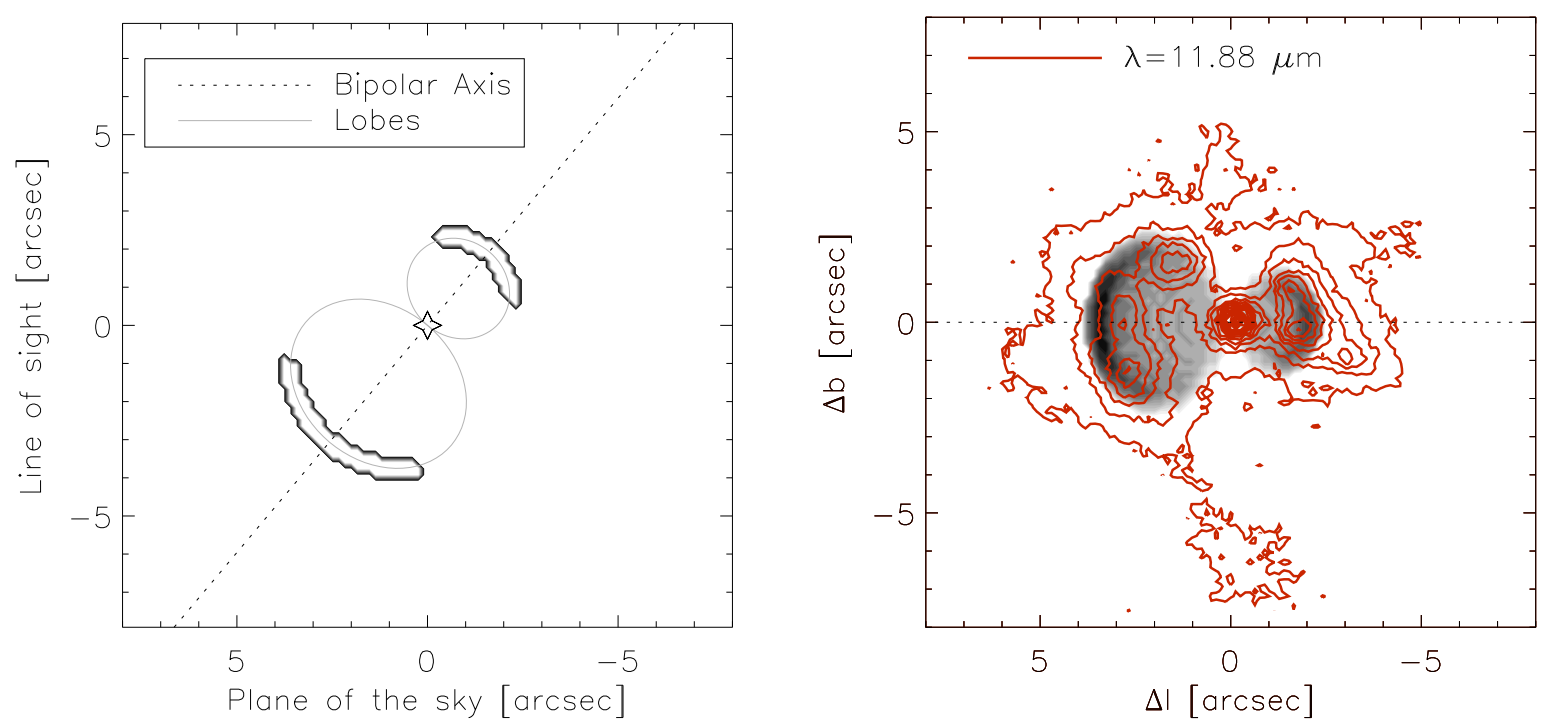

Figure 10. Schematic representation of the bipolar structure assumed in our simple geometrical model. The left panel sketches a top view of the two lobes originated from the star. The right panel shows the $11.88-\mu \mathrm{m}$ (red lines) image contours overlapping the simulated emission from the model.

most of the nebula, but steeper indices are found in the outer regions. Since the central star does not appear to produce a sufficient quantity of ionizing photons to keep the nebula ionized (Nota et al. 1997) and considered the local variation of the radio spectral index, it is possible to hypothesise that a fraction of the radio-emitting electron population comes from the shock of the stellar wind interacting with the denser surrounding envelope. This could result in the gradually steepening of the spectral index toward the edge of the mid-IR emission structures, where $\alpha \approx-0.6$ matches with what expected from synchrotron emission due to shock-accelerated electrons.

\subsection{The jet-precession model}

As shown in Figure 5, the radio images at 5 and $9 \mathrm{GHz}$ reveal that the ionized nebula is elongated, with a major axis at a position angle of about $25^{\circ}$ (North to East). The mid-IR images at 10.49 and $11.88 \mu \mathrm{m}$ show several blobs in an almost elliptical line around the central star, with the North-West features being closer to the star $(\sim 1.6 \mathrm{arcsec})$ than those in the South-East direction (more than 3 arcsec). The major axis of this pseudo-ellipse has a position angle of about $120^{\circ}$ and the minor axis of about $25^{\circ}$. It is worth to note that the elongation of the radio nebula and the minor axis of the pseudo-ellipse tracing the mid-IR features are almost identical. This suggests that the blobs visible in the mid-IR lie in a plane perpendicular to the axis of the radio nebula, in an almost circular region with centre offset with respect to the central star. A closer inspection of the mid-IR images reveals that the blobs are located in an Archimedean spiral centred on the star, where the distance $r$ to the blobs increases linearly with the polar angle $\theta$. Fig 11 illustrates the model. Adopting a distance of $5.4 \mathrm{kpc}$, the equation of the spiral is:

$$
r=r_{0}+\omega \times \theta
$$

with $r_{0}=1.6$ arcsec, corresponding to 8640 A.U., or $0.042 \mathrm{pc}$, and $\omega=0.46$ arcsec/radians.

As already reported, the circumstellar features observed in $\mathrm{H} \alpha$ have been interpreted by Nota et al. (1997) as a bipolar nebula with two lobes, with the symmetry axis oriented at an angle of $\approx 50^{\circ}$ (North to West). However, we note that these lobes are not complete, since the most evident features are an S-shaped emitting region centred on the star and a sort of elliptical diffuse emission elongated in the East-West direction (see Fig. 12). The straight line tangential to the S-shaped feature at the position of the star has the same inclination of about $25^{\circ}$ (North to East), coinciding with the major axis of the radio nebula and with the direction perpendicular to the plane where the "spiral" lies.

The idea is that of a jet-precession model, illustrated in Fig. 12. It is still a preliminary model that will be developed in more details in a subsequent paper. The stellar wind is collimated along the rotational axis of HR Car and gives rise to $\mathrm{H} \alpha$ and Bremsstrahlung emission, the latter being due to the ionization caused also by a hot companion, the B0 V star supposed by White (2000) in his symbiotic model. The presence of the hot companion in the East side can justify the values of the spectral index in the radio emission, as due to a wind-wind interaction. On the contrary, it is unlikely that the companion detected by Boffin et al. (2016) could contribute to the nebula ionization due to the low ionizing power (effective temperature) of red supergiants.

Violent episodes of copious mass loss occur in the equatorial plane when the stellar radius increases, approaching the equatorial rotational breakup velocity (Groh et al. 1999). These mass-loss events generate the blobs of the spiral visible in the near-IR. The direction of the ejection of the blobs may depend on the position of a companion star at the moment of the ejection. This star is probably either the B0 V or the closer massive red supergiant recently detected by Boffin et al. (2016) with the VLTI.

Although so far only one companion has been confirmed, if HR Car is a triple system, it is subject to a precession that could 

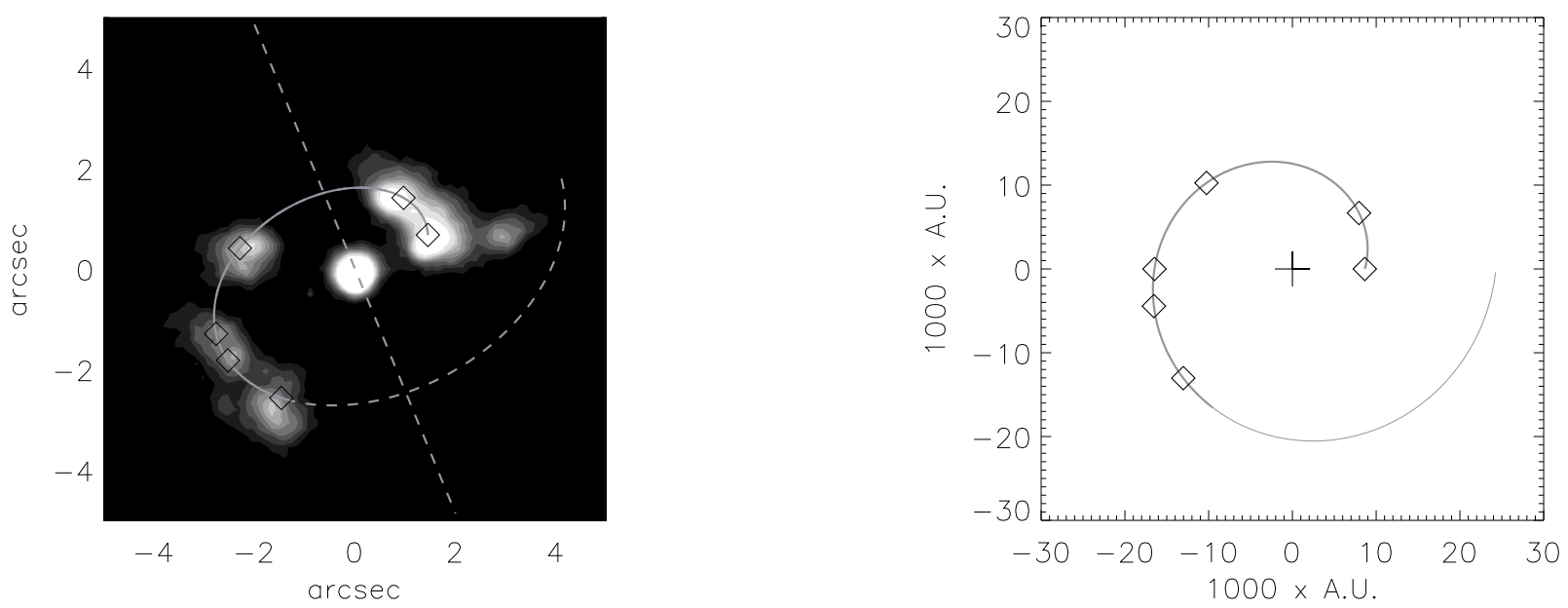

Figure 11. Left panel: the mid-IR image of HR Car at the SIV filter in the symbiotic model. The axis of the system (dashed line) is oriented $25^{\circ}$ counterclockwise. The blobs are located in an Archimedean spiral centred on the star. The plane where the spiral lies is inclined by $45^{\circ}$ relatively to the line of sight. Right panel: the same spiral seen pole on; symbols locate the observed blobs; distances are in A.U. adopting a distance to HR Car of 5.4 kpc

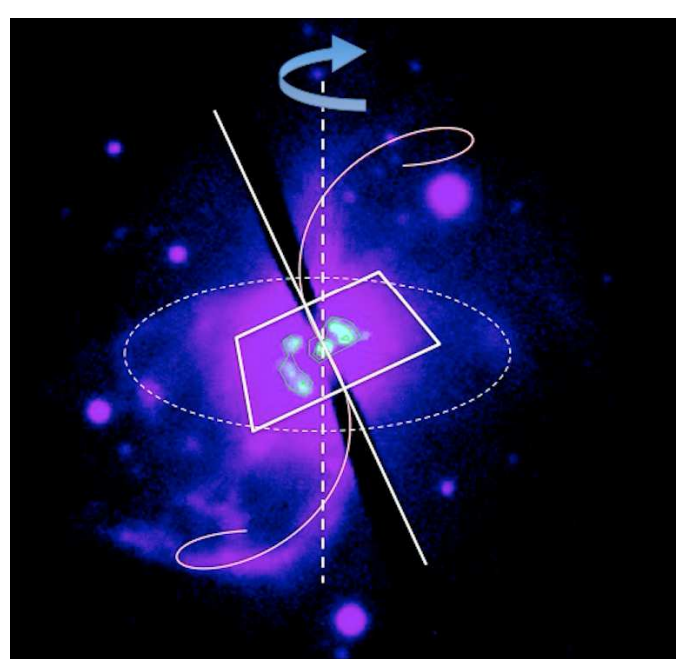

Figure 12. Schematic view of the jet-precession model for HR Car. The $\mathrm{H} \alpha$ image from archive show the S-shaped jet. Mid-IR blobs form a spiral lying in a plane (represented by a square drawn in perspective). The outflow visible in radio and $\mathrm{H} \alpha$ is perpendicular to this plane and precesses around an axis in North-South direction (dashed line). Diffuse $\mathrm{H} \alpha$ emission is confined in the shades ellipse.

give rise to a helical outflow observed as the S-shaped feature. The precession axis is approximately in the North-South direction. The matter ejected in the equatorial plane of the star over a time longer than the precession time accumulates in a circumstellar disc perpendicular to the precession axis. This is visible in the $\mathrm{H} \alpha$ image inside the ellipse of Fig. 12.

Note that hints for a jet-precession in the LBV star RMC127 in the Large Magellanic Cloud has been recently proposed by Agliozzo et al. (2016) and a precessing helical outflow has been observed in the Wolf-Rayet star WR102c by Lau et al. (2016).

\section{SUMMARY}

In this paper, we have presented a multi-wavelength study of the circumstellar environment surrounding HR Car, based on new midIR data obtained with VISIR-VLT and new radio data obtained with the ATCA. Such observations have been complemented by Herschel images from the Hi-GAL and MESS projects and with radio ATCA archive data. We have found that the nebular morphology varies greatly with the wavelength, confirming the presence of a complex multiple-shell structure.

The Herschel observations have revealed an extended envelope that reaches a distance of 70 arcsec from the central object toward NW. We derived a dust mass content of at least $1.6 \times 10^{-3} \mathrm{M}_{\odot}$ and, assuming a gas-to-dust ratio of 200 , a lower limit of $0.3 \mathrm{M}_{\odot}$ for the total nebular mass, which is consistent with the values previously reported in the literature. It is difficult to assess if the asymmetric distribution is the result of a density gradient in the ambient medium or it is caused by asymmetric mass-loss episodes that could be a consequence of binary interaction. We note that the location of the emitting structure and the derived dynamical age are consistent with a bow shock resulting from the interaction of the circumstellar material with the ISM.

At mid-infrared wavelengths, the VISIR images show two arcshaped structures surrounding the central star. These can be attributed either to the limb brightening of optically thin dust in a bipolar lobe-like structure or to an equatorially enhanced mass loss such as a spiral outflow arising from the binary interaction in the expanding wind of the mass-losing star. We discussed how the observed nebular morphology can be interpreted in the framework of the different proposed scenarios.

The new radio observations allowed us for the first time to clearly distinguish the central stellar wind from the nebular emission. We could then derive a mass-loss rate of $1.17 \times 10^{-5} \mathrm{M}_{\odot} \mathrm{yr}^{-1}$ in 2015. This value is 1.9 times larger than the mass-loss rate estimated in 1994-1995. This variation does not seem to be correlated to the S Dor cycle, but it could have been induced by the periastron passage of the companion star.

The brightest part of the ionized nebula lies on the opposite 
side of the bulk of the dust and seems to be well confined within the mid-IR structure. Finally, the comparison of our new radio data with the historic ATCA observations of HR Car at 9 and $18 \mathrm{GHz}$, which we reduced in a coherent way with the rest of our data, revealed significant changes in both the morphology of the nebula and the flux from the central star.

\section{ACKNOWLEDGMENTS}

This research is supported by ASI contract I/038/08/0 "HI-GAL". This work is based also on observations made with the VISIR instrument on the ESO VLT telescope (programme ID: 386.D0727A). The Australia Telescope Compact Array is part of the Australia Telescope National Facility which is funded by the Australian Government for operation as a National Facility managed by CSIRO. This paper includes archived data obtained through the Australia Telescope Online Archive (http://atoa.atnf.csiro.au).

CA acknowledges support from FONDECYT grant No. 3150463.

\section{REFERENCES}

Agliozzo C. et al, 2016, submitted to ApJ

Barlow M. J. et al., 2005, ApJL, 627, L113

Boffin H. M. J. et al., 2016, A\&A, 593, 90

Buemi C. S., Umana G., Trigilio C., Leto P., Hora J. L., 2010, ApJ, 721, 1404

Chiotellis A., Boumis, P., Nanouris N., Meaburn J., Dimitriadis G., 2016, MNRAS, 457, 9

Clampin M., Schulte-Ladbeck R. E., Nota A., Robberto M., Paresce F, Clayton G.C., 1995, AJ, 110, 251

Cox N. L. J., Kerschbaum F., van Marle A.-J., et al. 2012, A\&A, 537, A35

Duncan R. A., White S. M., 2002, MNRAS, 330, 63

Elia D., et al., 2013, ApJ, 772, 45

Frank A., Dongsu R., Davidson K, 1998, ApJ, 500, 291

Gvaramadze V. V., et al., 2015, MNRAS, 454, 219

Groenewegen M.A.T., Waelkens C., Barlow M.J. et al., 2011, A\&A, 526, A162

Groh J.H., Hillier D.J., Barbá R., Fernández-Lajús E., Gamen R.C., Moisés A.P., Solivella G., Teodoro M., 2009, ApJ, 705, L25

Groh J.H., Hillier D.J. and Damineli A., 2011, ApJ, 736, 46

Johnson D. R. H., Soderblom D. R., 1987, AJ, 93, 864

Jorissen A., et al., 2011, A\&A, 532, 135

Humphreys R. M., Davidson K., 1994, PASP, 106, 1025

Hutsemékers D., Van Drom E., 1991, A\&A, 248, 141

Kochanek C. S., 2011, ApJ, 743, 73

Kotak R., Vink J. S., 2006, A\&A, 460, L5

Lagage P.O., Pel J. W., Authier M., et al. 2004, The Messenger, 117, 12

Lamers H.J.G.L.M., Morris P.W., Voors R.H.M., et al., 1996, A\&A,315, 225

Lamers H.J.G.L.M., Nota A., Panagia N., Smith L. J., Langer N, 2001, ApJ, 551,764

Lamers H.J.G.L.M., Nugis T., 2002, A\&A, 395, L1

Lau R. M., Hankins M. J., Herter T. L., Morris M. R., Mills E. A. C. Ressler M. E, 2016, ApJ, 818, 117.

Leitherer C., Robert C., 1991, ApJ, 377, 6

Machado M. A. D., de Araújo F. X.., Pereira C. B., Fernandes M. B., 2002, A\&A, 387, 151

Maeder A., Desjacques V., 2001, A\&A, 372, L9

Mayer A., Deschamps R., Jorissen A., 2016, A\&A, 587, 30

Mihalas D., Binney J., 1981, in Mihalas D., Binney J., eds, Galactic Astronomy: Structure and Kinematics, 2nd edn., San Francisco, CA, W. H. Freeman and Co.

Molinari S., et al., 2010, PASP, 122, 314
Nota A., Livio M., Clampin M., Schulte-Ladbeck R., 1995, ApJ, 448,788

Nota A.,Smith L., Pasquali A., Clampin M., Stroud M., 1997, ApJ, 486,338

O’Hara T. B., Meixner M., Speck A. K., Ueta T., Bobrowsky M., 2003, ApJ, 598,1255

Ottensamer R., et al., 2011, in Why Galaxies Care about AGB Stars II: Shining Examples and Common Inhabitants, eds. F. Kerschbaum, T. Lebzelter, \& R. F. Wing, ASP Conf. Ser., 445, 625

Panagia N., Felli M., 1975, A\&A, 39, 1

Poglitsch A. et al., 2010, A\&A, 518, L2

Smith N., Owocki S. P., 2006, ApJ, 645, L42

Smith N., 2014, ARA\&A, 52, 487

Traficante A. et al., 2011, MNRAS, 416, 2932

Ueta T., et al., 2008, PASJ, 60, S407

Umana G., Buemi C.S., Trigilio C., Leto P., 2005, A\&A, 437, L1

Umana G., Buemi C.S., Trigilio C., Hora J.L., Fazio G.G., Leto P., 2009, ApJ, 694, 697

Umana G., Buemi C.S., Trigilio C., Leto P., Hora J.L., 2010, ApJ, 718, 1036 Vamvatira-Nakou C., Hutsemékers D., Royer P., Nazé Y., Magain P., Exster K., Waelkens C., Groenewegen M. A. T., 2013, A\&A, 557, 20

Vamvatira-Nakou C., Hutsemékers D., Royer P., Cox N. L. J., Nazé Y., Rauw G., Waelkens C., Groenewegen M. A. T., 2015, A\&A, 578, 108 van Genderen A. M., Robijn F. A. H., van Esch B. P.M., Lamers H. J. G. L. M., 1991, A\&A, 246, 4

van Genderen A. M., 2001, A\&A, 366, 508

van Leeuwen F., 2007, A\&A, 474, 653

Vink J. S., \& de Koter A., 2002, A\&A, 393, 543

Voors R.H.M., Waters L.B.F.M., Trams N.., Kaufl H.U., 1997, A\&A, 321, L21

Weis K., Duschl,W. J., Bomans, D. J., Chu, Y. H., \& Joner, M. D. 1997, A\&A, 320, 568

Weis K., 2001, Rev. Modern Astron., 14, 261

White S. M., 2000, ApJ, 539, 851

Wright E. L. et al., 2010, AJ, 140, 1868 